\title{
Predicting daily functioning with the modified Telephone Interview for Cognitive Status
}

\author{
Ava Dixon ${ }^{1} \cdot$ Sariah Porter ${ }^{1} \cdot$ Kayla Suhrie $^{1} \cdot$ Dustin Hammers $^{2} \cdot$ Kevin Duff $^{1}$ (i)
}

Received: 9 December 2021 / Accepted: 19 January 2022 / Published online: 11 February 2022

(c) The Author(s), under exclusive licence to Springer Nature Switzerland AG 2022

\begin{abstract}
Background The modified Telephone Interview for Cognitive Status (mTICS) is a frequently used telephone-based cognitive screening measure that can distinguish between normal aging, mild cognitive impairment (MCI), and dementia. Although it has been used to predict current and future cognitive function in older adults, no studies have examined if the mTICS can predict daily functioning.

Aims The current study sought to examine the relationship between the mTICS and a performance-based measure of daily functioning.

Methods The mTICS and demographic information (age, sex, education) were collected during a telephone screening visit for 149 older adults (65-91 years in age) with amnestic MCI. Three subscales of the Independent Living Scales (ILS; Managing Money, Managing Home and Transportation, Health and Safety) were collected during a baseline visit and during a 16 month follow-up visit in a subsample of 93 individuals.

Results Using simple hierarchical regression, baseline mTICS total score combined with demographic variables significantly predicted 19-22\% of baseline ILS subscale scores. Similarly, in a subsample of 93 participants with 16 month follow-up data, baseline mTICS and demographic information predicted 9-31\% of ILS subscale scores at follow-up.

Conclusions The mTICS appears able to predict daily functioning in older individuals with MCI. Remote tracking of cognition and daily functioning in this at-risk group seems particularly beneficial to geriatricians and other providers, especially during COVID-19.
\end{abstract}

Keywords Modified telephone interview for cognitive status $\cdot$ Mild cognitive impairment $\cdot$ Daily functioning

We certify that this work is novel and that it has the potential to enhance remote care of geriatric patients during the COVID-19 pandemic. Our findings demonstrate that a telephone-screening instrument for cognition can also be used to predict daily functioning at baseline and over time, which would allow researchers and clinicians to accurately and remotely track older adults' decline and risk of developing dementia when in-person evaluations are not feasible.

Kevin Duff

kevin.duff@hsc.utah.edu

1 Department of Neurology, Center for Alzheimer's Care, Imaging and Research, University of Utah, 650 Komas Drive \#106-A, Salt Lake City, UT 84108, USA

2 School of Medicine, Indiana University, Indianapolis, USA

\section{Introduction}

Dementia screening continues to be crucial for clinicians and researchers working with geriatric patients. In screening for dementia, individuals need to be evaluated for cognitive and functional decline, as both are core criteria for a diagnosis of Alzheimer's dementia [1,2]. Additionally, prompt screening is crucial to identify those at risk for neurodegenerative diseases in the earliest stages when treatments can be most effective [3]. However, in-person cognitive evaluations are becoming increasingly impractical as they are costly, timeconsuming, and difficult to provide to those with mobility issues or those living in rural areas [4]. With all of these limitations, telehealth is rapidly expanding to meet the needs of this patient population, which became significantly more acute during the COVID-19 pandemic [5].

One valuable addition to the telehealth toolbox is the modified Telephone Interview for Cognitive Status (mTICS) 
$[6,7]$, which allows for screening of multiple cognitive domains (e.g., memory, orientation, language) over the telephone. While similar global cognitive screening tools like the Mini Mental State Examination (MMSE) tend to be used more commonly, motor and visual components require face-to-face interaction. Researchers also found that such tasks limit a battery's ability to accurately measure cognitive performance in older adults for whom reading and drawing are difficult [8]. To address this, Moylan and colleagues [8] found significant correlations between mTICS and MMSE scores making the mTICS a reliable alternative whose measurement of cognitive impairment is not biased by physical limitations. Researchers have also found that the mTICS is more strongly correlated with and more strongly predicts performance at baseline and one year later on a larger, memory-based neuropsychological battery than the MMSE [9]. Multiple studies have demonstrated the ability of the mTICS to distinguish intact older adults from those with Mild Cognitive Impairment (MCI) and dementia [10-13]. Additionally, researchers have developed regression-based models using the mTICS and demographic variables to predict a composite score from multiple memory tests at baseline and 1 year follow-up [13]. Such prediction equations could be used in clinical or research settings to remotely estimate performance on an in-person assessment of memory.

While multiple studies have observed how telephone screening instruments like the mTICS predict cognition, few have examined the relationship between these screening scales and functional abilities (e.g., handling money, managing medications, completing chores around the home). The measure of daily functioning is crucial to the diagnosis of dementia, therefore relating remote screening measures to functional abilities would be highly advantageous. For example, Schmitter-Edgecombe and colleagues [14] found significant correlations between scores on the Telephone Interview for Cognitive Status (TICS) and scores on multiple functional assessments. McGuire, Ford, and Ajani [15] determined that scores on an adapted TICS significantly predicted self-reported scores on an instrumental activities of daily living scale. To our knowledge, no studies have examined the relationship between the mTICS and measures of functional ability. The mTICS builds on the original TICS by including a delayed memory component, which increased the difficulty of the mTICS and improved its ability to identify early indicators of dementia [7]. As impaired daily functioning is the distinguishing factor between those with dementia or MCI, studies relating the mTICS to functional abilities are needed. Given its greater sensitivity to differentiate between normal aging, MCI, and dementia based on cognitive performance [10-13], the mTICS may be wellequipped to predict functional performance.

In response to this need, the current study sought to further examine the relationship between mTICS and functional abilities in individuals with amnestic MCI. The first aim of the study focused on the ability of the mTICS to predict baseline scores on a performance-based measure of instrumental activities of daily living. It was hypothesized that the total score of the mTICS would significantly predict an individual's current functional abilities. Additionally, we extended these prediction equations to examine how the mTICS could predict scores on a performance-based measure of IADLs at a later, follow-up visit. Similarly, it was hypothesized that the total score of the mTICS would significantly predict future functional abilities. Using the mTICS to predict future daily functioning may identify those at risk of developing dementia over the following 1-1.5 years. As a third aim, age, education, and sex were added to both prediction equations. Such demographic information is easily obtainable, and as previous studies have demonstrated significant correlations between demographics and performance of daily tasks [16-18], it was expected that the addition of such variables would enhance the prediction equations. Based on previous findings, it was hypothesized that age would negatively affect the prediction of daily functioning, education would positively affect this prediction, and sex would have little to no effect on it.

\section{Methods}

\section{Participants}

One hundred and forty-nine community-dwelling older adults were recruited through a cognitive disorders' clinic, independent living facilities, and community senior centers to participate in a cognitive intervention study. Their mean age was $75.4(\mathrm{SD}=6.1$, range $=65-91)$ years and they averaged 16.2 $(\mathrm{SD}=2.9$, range $=12-25)$ years of education. The sample was evenly divided by sex ( $49.0 \%$ female) and nearly all were Caucasian (98.7\%). For inclusion in the study, all participants from this sample were classified as having either single-domain or multi-domain amnestic MCI based on the core clinical criteria outlined by Albert and colleagues [19], including memory complaint, objective memory deficit, and largely intact daily functioning based on participant and collateral report. Classification of participants from this sample has been described previously [20]. General inclusion criteria for the study involved being aged 65 years or older and functionally independent (according to the participant and/ or knowledgeable informant), along with adequate vision, hearing, and motor abilities to complete the cognitive evaluation. General exclusion criteria included neurological conditions likely to negatively affect cognition (e.g., stroke, seizures, traumatic brain injury with loss of consciousness of more than $30 \mathrm{~min}$ ), dementia, major psychiatric conditions 
(including current severe depression), substance abuse, or residency in a skilled nursing or dependent living facility.

Participants were compensated for their time for the cognitive and functional assessments. Prior to enrollment, participants' cognitive abilities were screened over the telephone using the mTICS. Based on cutoff scores from Lines and colleagues [21], individuals who received a total score below 20 were excluded as it was suggestive of dementia, and those who received a score no greater than 8 out of 20 (total score $\leq 38$ ) on the combined immediate and delayed recall portion were considered likely to meet criteria for amnestic MCI. Participants with a clinical diagnosis of MCI were included regardless of their combined immediate and delayed recall scores. With these exceptions, the upper range of the mTICS total score was 42 .

All procedures were approved by the local institutional review board and written informed consent was obtained from participants before study procedures commenced.

\section{Materials}

\section{Telephone interview for cognitive status-modified}

The mTICS $[6,7]$ is a 14 -item telephone screening instrument that assesses global cognition, with an emphasis on learning and memory performed before enrollment in the study. The total range is $0-50$, with higher scores indicating better cognition. Similar to other cognitive screening measures like the Mini-Mental State Examination, the mTICS assesses orientation (e.g., participant's name, telephone number, month, date, year, season, day of the week; 9 points), attention (e.g., counting backwards, serial sevens; 7 points), and language (e.g., naming, word opposites, phrase repetition, following simple commands; 14 points). This measure also emphasizes new learning and memory with immediate and delayed recall of a 10-item word list. (10 points each). Given the total score on this measure is weighted to memory (20 out of 50 points), this instrument might be particularly useful in identifying cases of early dementia and amnestic MCI. The total score on the mTICS was the primary independent variable for this measure.

\section{Independent living scales}

The ILS [22] is an individually administered objective assessment of daily functioning for older adults that was performed at baseline and follow-up. It is a reliable and valid standardized measure for identifying areas of competence in five subscales: Memory/Orientation, Managing Money, Managing Home and Transportation, Health and Safety, and Social Adjustment. The ILS was standardized using a nationally-stratified sample of 400 older adults (age $\geq 65$ years) with no known cognitive or functional deficits. For this project, only the Managing Money, Managing Home and Transportation, and Health and Safety subscales were utilized, as the other two subscales of the ILS seemed either redundant with the mTICS (Memory/Orientation) or less relevant to the study (Social Adjustment). For the Managing Money subscale, individuals were asked practical questions about money (e.g., how much common items cost at a grocery store, counting out exact change, performing simple arithmetic by hand, writing checks, knowing the purpose of a will). For the Managing Home and Transportation subscale, questions and tasks addressed issues around the home and traveling into the community (e.g., how to take public transportation, identifying routine tasks performed at home, looking someone up in a telephone book and calling that number, demonstrating how to call the operator). For the Health and Safety subscale, participants are asked about health and health emergencies (e.g., how to notify officials in health and safety emergencies, explain how to safely cross a busy street, know precautions to take when going out at night, explain how to practice correct personal hygiene). Using normative data from the standardization sample, raw scores were converted to T-scores $(M=50, \mathrm{SD}=10)$ for each subscale, with higher scores indicating better functional abilities. No demographic corrections (e.g., age, education, sex) are provided in the normative data. These three subscale $\mathrm{T}$-scores were the primary dependent variables from this measure.

\section{Procedures}

Before enrollment, participants completed a 10 min telephone screening at which the mTICS was collected. On enrollment, participants completed the Independent Living Scales (ILS) which lasted $1 \mathrm{~h}$ in a $3.5 \mathrm{~h}$ battery at a baseline visit which on average took place $23(\mathrm{SD}=20.5$, range $=1-128$ ) days after the screening. A subset of the baseline sample $(n=93)$ completed the ILS again as part of the overall battery approximately $16(\mathrm{SD}=1.7$, range $=10.6-21.4$ ) months later at a follow-up visit scheduled one year after participants completed the cognitive intervention which did not significantly improve cognitive or functional outcomes [23].

\section{Data analyses}

Descriptive statistics examined the means and standard deviations of the mTICS total score, as well as the three subscales of the ILS in this MCI sample at baseline and followup and correlations were also calculated. Correlations were also performed to evaluate the relationship between mTICS total scores, age, education, and ILS subscale T-scores. To measure differences for sex, independent sample $t$ tests were performed. Simple hierarchical regressions were performed 
to (1) evaluate the relationship between the three ILS subscales and the total score on the mTICS and (2) examine how demographics (age, education, and sex) enhanced those predictions by comparing $R^{2}$ change scores. ILS subscale T-score acted as the dependent variable and mTICS total score and demographic variables acted as predictor variables. Two sets of regression models were calculated, one using baseline ILS T-scores and another using 16 month follow-up ILS T-scores. An alpha value of $p<0.05$ was utilized. All statistical analyses were performed using SPSS statistics software.

\section{Results}

\section{Descriptive information on MTICS and ILS subscales}

During the telephone screening, participants scored an average of $31.17(\mathrm{SD}=4.38$, range $=20-42)$ on the mTICS. At a baseline in-person visit, participants' mean T-score fell within the average range, although there was some variability, on the ILS Managing Money $(M=50.91$, $\mathrm{SD}=9.13$, range $=24-61)$, Managing Home and Transportation $(M=50.42, \mathrm{SD}=6.26$, range $=23-59)$, and Health and Safety $(M=51.49, \mathrm{SD}=7.62$, range $=20-63)$ subscales. At the 16-month follow-up, participants continued to exhibit mean $\mathrm{T}$-scores within the average range on the Managing Money, $(M=50.70, \mathrm{SD}=9.20$, range $=20-61)$, Managing Home and Transportation $(M=49.58, \mathrm{SD}=7.82$, range $=20-59)$, and Health and Safety $(M=51.38$, $\mathrm{SD}=9.43$, range $=20-63$ ) ILS subscales.

\section{Demographic effects on mTICS and ILS subscales}

Correlations revealed that age was only significantly related to the Health and Safety subscale at baseline and followup and education was significantly related to mTICS total scores, and baseline T-scores for Managing Home and Transportation and Health and Safety. Full correlations are provided below in Table 1. Independent sample $t$ tests demonstrated equal variance between males and females for mTICS total scores and T-scores for the baseline Managing
Money and Transportation and follow-up Managing Money ILS subscales. Men scored higher on ILS Managing Money at baseline $(M=52.25, \mathrm{SD}=7.90)$ than women $(M=49.52$, $\mathrm{SD}=10.12), t(147)=-1.83, p=0.069$. Significant differences were also seen on the Home and Transportation Subscale at follow-up with men $(M=51.12, \mathrm{SD}=5.91)$ scoring above women $(M=47.71, \mathrm{SD}=9.39), t(91)=-2.04$, $p=0.045$. For Health and Safety at baseline men $(M=52.76$, $\mathrm{SD}=6.84)$ also scored higher than women $(M=50.16$, $\mathrm{SD}=8.19), t(147)=-2.10, p=0.038$. At follow-up, men $(M=53.49, \mathrm{SD}=7.04)$ continued to score higher than women $(M=48.76, \mathrm{SD}=11.28)$ on the Health and Safety subscale, $t(90)=-2.34, p=0.022$.

\section{Predicting baseline ILS with mTICS}

The mTICS total score significantly predicted T-scores for all three ILS subscales at baseline. It significantly predicted performance on the Managing Money subscale, $F$ $(1148)=38.5, p<0.001, R^{2}=0.21$. The Managing Home and Transportation subscale was significantly predicted by mTICS total score, $F(1148)=27.5, p<0.001, R^{2}=0.16$. Finally, mTICS total score significantly predicted the Health and Safety subscale, $F(1148)=33.8, p<0.001, R^{2}=0.19$. Constant and unstandardized beta weights from these equations are presented in Table 2. Anticipating an $R^{2}$ of 0.15 , it was determined that to obtain a power of at least $80 \%$ when $p<0.05$, the sample size would need to include a minimum of 50 subjects if the mTICS score was the only predictor variable. Given that all observed $R^{2}$ exceed 0.15 , these analyses are adequately powered.

\section{Predicting baseline ILS with mTICS and demographic variables}

Upon adding demographic variables, the mTICS total score continued to significantly predict performance on all subscales of the ILS, however, the addition of demographic variables did not significantly improve the equations. The Managing Money subscale, $F(4148)=10.3, p<0.001, R^{2}=0.22$, Sig $F$. Change $=0.45$. The Managing Home and Transportation subscale, $F(4148)=8.6, p<0.001, R^{2}=0.19$, Sig $F$.

Table 1 Correlation between demographic variables, ILS subscales, and mTICS total score

\begin{tabular}{llllllll}
\hline & Baseline MM & Baseline MHT & Baseline HS & Follow-up MM & Follow-up MHT & Follow-up HS & mTICS \\
\hline Age & -0.16 & -0.11 & $-0.20^{\mathrm{a}}$ & -0.10 & -0.003 & $-0.26^{\mathrm{a}}$ & -0.19 \\
Education & 0.16 & $0.29^{\mathrm{b}}$ & $0.23^{\mathrm{b}}$ & 0.18 & 0.13 & 0.08 & $0.29^{\mathrm{b}}$ \\
\hline
\end{tabular}

ILS Independent Living Scales, MM Managing Money, MHT Managing Home and Transportation, HS Health and Safety; age and education are in years; mTICS is raw total score

${ }^{\mathrm{a}}$ Correlation significant at 0.05

${ }^{\mathrm{b}}$ Correlation is significant at 0.01 
Table 2 Predicting ILS subscale T-scores from mTICS total score

\begin{tabular}{lllll}
\hline ILS subscale & $F(\mathrm{df})$ & $R^{2}$ & $\mathrm{SE}_{\mathrm{est}}$ & Prediction equation \\
\hline Baseline managing money & $38.5(1148)$ & 0.21 & 8.15 & $21.30+(\mathrm{mTICS} \times 0.95)$ \\
Baseline managing home and transportation & $27.5(1148)$ & 0.16 & 5.76 & $32.75+(\mathrm{mTICS} \times 0.57)$ \\
Baseline health and safety & $33.8(1148)$ & 0.19 & 6.89 & $28.05+(\mathrm{mTICS} \times 0.75)$ \\
Follow-up managing money & $34.6(192)$ & 0.28 & 7.88 & $13.30+(\mathrm{mTICS} \times 1.19)$ \\
Follow-up managing home and transportation & $5.1(192)$ & 0.05 & 7.65 & $35.62+(\mathrm{mTICS} \times 0.44)$ \\
Follow-up health and safety & $10.3(191)$ & 0.10 & 8.98 & $27.68+(\mathrm{mTICS} \times 0.75)$ \\
\hline
\end{tabular}

ILS Independent Living Scales, $S E_{\text {est }}$ Standard error of the estimate, age is in years; education is in years; sex is coded as $0=$ female, $1=$ male; mTICS is the raw total score. All prediction equations were significant, $p<0.001$ for all subscales except for Managing Home and Transportation at follow-up, $p=0.03$ and Health and Safety at follow-up, $p=0.002$

Change $=0.10$. Finally, the Health and Safety subscale, $F$ $(4148)=10.3, p<0.001, R^{2}=0.22$, Sig $F$. Change $=0.09$. Constant and unstandardized beta weights from these equations are presented in Table 3.

\section{Predicting 16-month ILS with mTICS}

The Managing Money subscale at 16 month follow-up was significantly predicted by mTICS total score, $F(192)=34.6$, $p<0.001, R^{2}=0.28$. The Managing Home and Transportation subscale was significantly predicted by mTICS total score, $F(192)=5.1, p=0.03, R^{2}=0.05$. Finally, mTICS total score significantly predicted the Health and Safety subscale, $F(191)=10.3, p=0.002, R^{2}=0.10$. Constant and unstandardized beta weights from these equations are presented in Table 2. Using the same power analyses described above, these analyses were adequately powered except for the Managing Home and Transportation equation, which only had a power of $60 \%$.

\section{Predicting 16-month ILS with mTICS and demographic variables}

The Managing Money subscale at 16 month follow-up was significantly predicted by mTICS and demographic variables though demographic variables did not significantly improve equations, $F(4,92)=9.8, p<0.001, R^{2}=0.31$, $\mathrm{Sig}$ $F$. Change $=0.25$. Upon the addition of demographic variables, the Managing Home and Transportation subscale was no longer significantly predicted by mTICS total score and demographics, $F(4,92)=2.1, p=0.09, R^{2}=0.09$, Sig $F$. Change $=0.35$. Again, demographics did not significantly improve the equation. Finally, mTICS total score and demographics significantly predicted the Health and Safety subscale with this being the only subscale that demographics significantly improved, $F(491)=4.8$, $p=0.002, R^{2}=0.18$, Sig $F$. Change $=0.05$. Constant and unstandardized beta weights from these equations are presented in Table 3.

Table 3 Predicting ILS subscale T-scores from mTICS total score and demographic variables

\begin{tabular}{|c|c|c|c|c|}
\hline ILS subscale & $F(\mathrm{df})$ & $R^{2}$ & $\mathrm{SE}_{\text {est }}$ & Prediction equation \\
\hline Baseline managing money & $10.3(4148)$ & 0.22 & 8.16 & $\begin{array}{l}31.08+(\mathrm{mTICS} \times 0.90)-(\text { age } \times 0.12)+(\mathrm{sex} \times 1.44)+(\text { education } \\
\times 0.00)\end{array}$ \\
\hline Baseline managing home and transportation & $8.6(4148)$ & 0.19 & 5.70 & $\begin{array}{l}32.66+(\mathrm{mTICS} \times 0.48)-(\text { age } \times 0.05)-(\mathrm{sex} \times 0.01)+(\text { education } \times \\
0.41)\end{array}$ \\
\hline Baseline health and safety & $10.3(4148)$ & 0.22 & 6.81 & $\begin{array}{l}38.63+(\mathrm{mTICS} \times 0.66)-(\text { age } \times 0.16)+(\mathrm{sex} \times 1.09)+(\text { education } \\
\times 0.23)\end{array}$ \\
\hline Follow-up managing money & $9.8(492)$ & 0.31 & 7.83 & $\begin{array}{l}16.41+(\mathrm{mTICS} \times 1.11)-(\text { age } \times 0.02)+(\mathrm{sex} \times 3.43)-(\text { education } \times \\
0.06)\end{array}$ \\
\hline Follow-up managing home and transportation & $2.1(492)$ & 0.09 & 7.64 & $\begin{array}{l}30.06+(\mathrm{mTICS} \times 0.38)+(\text { age } \times 0.07)+(\mathrm{sex} \times 2.90)+(\text { education } \\
\times 0.04)\end{array}$ \\
\hline Follow-up health and safety & $4.8(491)$ & 0.18 & 8.72 & $\begin{array}{l}54.99+(\mathrm{mTICS} \times 0.66)-(\text { age } \times 0.30)+(\operatorname{sex} \times 3.49)-(\text { education } \times \\
0.24)\end{array}$ \\
\hline
\end{tabular}

Age is in years; education is in years; sex is coded as $0=$ female, $1=$ male; mTICS is the raw total score. All prediction equations were significant, $p<0.001$ for all subscales except for Health and Safety at follow-up, $p=0.002$. Prediction for Managing Home and Transportation at follow-up was no longer significant, $p=0.09$

$I L S$ Independent Living Scales, $S E_{\text {est }}$ Standard error of the estimate 


\section{Discussion}

Previous research has demonstrated that the mTICS can distinguish between normal aging, MCI, and dementia [10-13], and it has also been used to predict performance on more comprehensive cognitive batteries [9]. However, cognition is not the sole factor in a diagnosis of dementia, and daily functional abilities must also be considered. Hypothesizing that the mTICS would significantly predict T-scores of the ILS and that demographic variables would enhance these prediction equations, the current study expanded on the ability of the mTICS to remotely classify neurocognitive disorders using this screening measure to predict the performance of instrumental activities of daily living. Similar to the findings of Duff and colleagues [13], which demonstrated that the mTICS could predict performance on a neuropsychological battery at baseline and follow-up, this study found that the mTICS can also predict performance on an objective assessment of daily function at baseline and 16 months later.

In predicting baseline functional abilities, the mTICS total score alone accounted for 19-21\% (including demographics, 19-22\%) of the variance in the three ILS subscales. While few studies have examined the relationship between the mTICS and daily functional abilities, these results are consistent with the related literature. For example, Schmitter-Edgecombe and colleagues [14] found that TICS scores were significantly correlated with performance on multiple objective measures of daily function in cognitively healthy older adults. Additionally, another telephone-based screening instrument, the Minnesota Cognitive Acuity Screen, significantly predicted daily functioning as measured by a subjective report in older adults with MCI [24]. The results of the current study extend the applicability of the mTICS to not only predict scores on comprehensive in-person cognitive batteries but also scores on measures of functional abilities.

Similar to the baseline ILS predictions, the current study demonstrated that the mTICS could significantly predict performances on the ILS after 16 months. Overall, these models accounted for 5-28\% (including demographics, 9-31\%) of the ILS subscale variance after 16 months. This is consistent with findings that the TICS, combined with demographic variables and overall health, significantly predicted self-reported functional abilities of older adults without cognitive impairments 2 years after its administration [15]. More specifically, the TICS significantly predicted self-reported performance when it came to preparing meals, shopping, managing money, using a telephone, light housework, and managing medications. The ILS subscales used in the current study measure the same tasks through performance-based evaluation rather than the self-report used by McGuire and colleagues. Regardless of the method used to measure function, the results of McGuire et al. and the current study indicate that telephone-based screenings can be used to determine the current and future performance of independent activities of daily living.

Despite significant correlations between $\mathrm{T}$-scores of the ILS subscales and demographic variables, the addition of age, sex, and education did not significantly improve most predictions from those using mTICS total score alone. The only exception was that for Health and Safety at follow-up. Nonetheless, consistent with previous findings, age was negatively correlated with the ILS subscales and negatively weighted in all but one (Managing Home and Transportation at follow-up) of the regression equations [16-18]. Similar to findings from Duff et al. [25], age was most strongly correlated with the Health and Safety subtest of the ILS at baseline and follow-up. As hypothesized, education was positively correlated with all subscales of the ILS, which is consistent with previous findings [17, 18]. Correlations for education were only significant for the baseline Managing Home and Transportation and Health and Safety subscales. Despite the significant differences between men and women on Managing Home and Transportation at follow-up and Health and Safety at baseline and follow-up, sex did not significantly improve regression models. This is consistent with previous findings [17, 24]. Even though demographic variables did not notably improve the prediction of daily functioning with the mTICS, such demographic information is easily obtainable, these variables are frequently related to cognition, and previous studies have demonstrated relationships between demographics and performance of daily tasks [16-18]. As such, it seemed important to at least consider them in our analyses.

Case examples may better illustrate the application of these prediction models. A 70 year-old potential research participant with 16 years of education receives a total score on the mTICS of 31. Using the values in Table 2, an investigator could predict that this individual's Managing Home and Transportation T-score at baseline would be approximately 50 (i.e., $32.75+[31 \times 0.57]=50.42$ ). If the investigator were looking for subjects without functional impairment, this particular individual may be more likely to meet inclusion criteria. The prediction models could also be used to remotely predict functional decline in older patients who are unable to come into the clinic. For example, if an 89 year-old female with 20 years of education scores 23 on the mTICS at baseline, using the equations from Table 3, clinicians could predict that her Health and Safety T-score would be 44 at baseline (i.e., $38.63+[23 \times 0.66]-[89 \times 0.16]+[0 \times$ $1.09]+[20 \times 0.23]=44.17)$ and 39 at 16 month follow-up (i.e., $54.99+[23 \times 0.66]-[89 \times 0.30]+[0 \times 3.49]-[20 \times$ $0.24]=38.67$ ). This would indicate a decline in her daily 
functioning from just over one half of a standard deviation at baseline to over a full standard deviation below expectations at follow-up. This particular individual might require more services than someone who is expected to stay functionally stable over the next year and a half. Furthermore, these equations allow clinicians and researchers to compare an individual's observed functional performance to his/her predicted functional scores. To measure how much an individual's observed ILS score differed from their predicted ILS score in z-score units, one would subtract the predicted $(p)$ score from the observed $(o)$ score and divide by the standard error of the estimate in Tables 2 or 3 (i.e., $z=\left[\mathrm{ILS}_{\mathrm{o}}-\mathrm{ILS}_{\mathrm{p}}\right] / \mathrm{SE}_{\mathrm{est}}$ ). For example, if the 70-year-old research participant's observed Managing Home and Transportation T-score was 40 and the predicted Managing Home and Transportation T-score was 50.42 , then their $z=([40-50.42] / 5.76)=-1.81$. Typically, a T-score of 40 denotes a single standard deviation below the mean; however, when using the predicted values, the observed functional abilities fall nearly two standard deviations below expectation for this individual. If the 89-year-old patient's observed baseline Health and Safety T-score was 45 and her predicted baseline Health and Safety T-score was 44.17 , then her $z=([45-44.17] / 6.81)=0.12$, which indicates that the patient's observed ILS score is nearly identical to her predicted ILS score. While these equations would need to be validated in an independent sample, they demonstrate that the mTICS is a valuable resource in identifying present and future functional abilities to aid in clinical and research settings. Readers may contact the corresponding author for a copy of the spreadsheet that will do these calculations.

While these findings have potential utility for clinicians and researchers, some limitations of the current study should be acknowledged. First, the homogenous study sample limits the generalizability of these current findings. The 149 participants in the baseline sample were almost exclusively Caucasian and highly educated. Future studies should strive to represent individuals of all races and ethnicities, socioeconomic classes, and educational levels to ensure that results are applicable to the rapidly diversifying society. Second, since this study focused on individuals with amnestic MCI, the range of scores on the mTICS and ILS were restricted, which could also limit the generalizability of these prediction equations. While individuals with MCI are diagnostically considered functionally intact, some studies suggest that their ability to perform instrumental activities of daily living is worse than their cognitively healthy peers [26]. Therefore, additional data from cognitively intact individuals and those with dementia would expand the applicability of the mTICS in predicting daily functioning. Third, this study only examined the ILS - a performance-based measure of functional abilities. Future studies might examine the relationship between the mTICS and self-/informantreported functional measures. Fourth, the mTICS combined with demographic variables predicted between 9 and $31 \%$ of the baseline and 16 month ILS scores. As the variance accounted for by these prediction models is relatively low, future studies should strive to identify other variables that improve these prediction models. Finally, the ILS used in the current study views functional abilities as both continuous (i.e., range of performances from severely impaired to well above average) and relative (i.e., different cutoffs depending on expectations of the individual), whereas clinical practice traditionally views functional abilities as both dichotomous (i.e., impaired/intact) and absolute (i.e., same cutoff for all). Neither of these views are inherently correct nor incorrect, but they can be challenging to use contemporaneously. Despite these limitations, the current findings remain beneficial, especially as telephone-based assessment and other forms of remote care are becoming increasingly necessary in society and proved even more crucial during the COVID-19 pandemic. Administration of this screening requires minimal training, takes less than $10 \mathrm{~min}$, and can be completed remotely, yet despite its simplicity, it is able to significantly predict both cognitive and functional performance in older adults. As such, it could be employed in primary care clinics to evaluate and track older patients to gauge their risk for dementia (e.g., cognitive and functional impairments) to decide if additional services are needed. Researchers might also use the mTICS to discern if older adults would be appropriate candidates (e.g., MCI or dementia) for research studies before bringing them into the lab. The ability of the mTICS to screen for both cognitive and functional impairment make it an indispensable tool in the classification of neurodegenerative disorders.

Author contributions All authors contributed to the study concept and design, acquisition of subjects and/or data, analysis and interpretation of data, and preparation of the manuscript.

Funding The project described was supported by research grants from the National Institute on Aging: R01AG045163. The content is solely the responsibility of the authors and does not necessarily represent the official views of the National Institute on Aging or the National Institutes of Health. This project also utilized REDCap, which is supported by 8UL1TR000105 (formerly UL1RR025764) NCATS/NIH.

Availability of data and material The datasets generated and analyzed during the current study are available from the corresponding author on reasonable request.

Code availability Not applicable.

\section{Declarations}

Conflicts of interest The authors have no conflicts of interest to declare. 
Ethical approval Approval was obtained from the Institutional Review Board of the University of Utah. The procedures used in this study adhere to the tenets of the Declaration of Helsinki.

Consent to participate Informed consent was obtained from all individuals included in this study.

Consent for publication Not applicable. Case reports are hypothetical and not representative of any individual participant in the study.

\section{References}

1. McKhann GM, Knopman DS, Chertkow H et al (2011) The diagnosis of dementia due to Alzheimer's disease: recommendations from the National Institute on Aging-Alzheimer's association workgroups on diagnostic guidelines for Alzheimer's disease. Alzheimers Dement 7:263-269

2. Atri A (2019) The Alzheimer's disease clinical spectrum: diagnosis and management. Med Clin N Am 103:263-293

3. Borson S, Frank L, Bayley PJ et al (2013) Improving dementia care: the role of screening and detection of cognitive impairment. Alzheimers Dement 9:151-159

4. Kirk Wiese L, Williams CL, Tappen RM (2014) Analysis of barriers to cognitive screening in rural populations in the United States. ANS Adv Nurs Sci 37:327-339

5. Hammers DB, Stolwyk R, Harder L et al (2020) A survey of international clinical teleneuropsychology service provision prior to and in the context of COVID-19. Clinic Neuropsychol 34:1267-1283

6. Brandt J, Spencer M, Folstein M (1988) The telephone interview for cognitive status. Neuropsychiatry Neuropsychol Behav Neurol 1:111-117

7. Welsh K, Breitner J, Magruder-Habib K (1993) Detection of dementia in the elderly using telephone screening of cognitive status. Neuropsychiatry Neuropsychol Behav Neurol 6:103-110

8. Moylan T, Das K, Gibb A et al (2004) Assessment of cognitive function in older hospital inpatients: is the Telephone Interview for Cognitive Status (TICS-M) a useful alternative to the Mini Mental State Examination? Int J Geriatr Psychiatry 19:1008-1009

9. Duff K, Tometich D, Dennett K (2015) The modified telephone interview for cognitive status is more predictive of memory abilities than the mini-mental state examination. J Geriatr Psychiatry Neurol 28:193-197

10. Yaari R, Fleisher AS, Gamst AC et al (2006) Utility of the telephone interview for cognitive status for enrollment in clinical trials. Alzheimers Dement 2:104-109

11. Cook SE, Marsiske M, McCoy KJ (2009) The use of the modified telephone interview for cognitive status (TICS-M) in the detection of amnestic mild cognitive impairment. J Geriatr Psychiatry Neurol 22:103-109

12. Knopman DS, Roberts RO, Geda YE et al (2010) Validation of the telephone interview for cognitive status-modified in subjects with normal cognition, mild cognitive impairment, or dementia. Neuroepidemiology 34:34-42
13. Duff K, Beglinger LJ, Adams WH (2009) Validation of the modified telephone interview for cognitive status in amnestic mild cognitive impairment and intact elders. Alzheimer Dis Assoc Disord 23:38-43

14. Schmitter-Edgecombe M, Parsey C, Cook DJ (2011) Cognitive correlates of functional performance in older adults: comparison of self-report, direct observation, and performance-based measures. J Int Neuropsychol Soc 17:853-864

15. McGuire LC, Ford ES, Ajani UA (2006) Cognitive functioning as a predictor of functional disability in later life. Am J Geriatr Psychiatry 14:36-42

16. Ahmed FS, Miller LS (2015) Adequate proverb interpretation is associated with performance on the independent living scales. Neuropsychol Dev Cogn B Aging Neuropsychol Cogn 22:376-387

17. Baird A (2006) Fine tuning recommendations for older adults with memory complaints: using the independent living scales with the dementia rating scale. Clin Neuropsychol 20:649-661

18. Emmert NA, Schwarz LR, Vander Wal JS et al (2019) Neuropsychological predictors of health and safety abilities in dementia. Appl Neuropsychol Adult 28:94-106

19. Albert MS, DeKosky ST, Dickson D et al (2011) The diagnosis of mild cognitive impairment due to Alzheimer's disease: recommendations from the national institute on Aging-Alzheimer's association workgroups on diagnostic guidelines for Alzheimer's disease. Alzheimers Dement 7:270-279

20. Duff K, Atkinson TJ, Suhrie KR et al (2017) Short-term practice effects in mild cognitive impairment: evaluating different methods of change. J Clin Exp Neuropsychol 39:396-407

21. Lines CR, McCarroll KA, Lipton RB et al (2003) Telephone screening for amnestic mild cognitive impairment. Neurology 60:261-266

22. Loeb PA (1996) Independent living scales manual. Psychological Corporation, San Antonio

23. Duff K, Ying J, Suhrie KR et al (2021) Computerized cognitive training in amnestic mild cognitive impairment: a randomized clinical trial. J Geriatr Psychiatry Neurol. https://doi.org/10.1177/ 08919887211006472

24. Tremont G, Papandonatos GD, Kelley P et al (2016) Prediction of cognitive and functional decline using the telephone-administered minnesota cognitive acuity screen. J Am Geriatr Soc 64:608-613

25. Duff K, Porter S, Dixon A et al (2020) The independent living scale in amnestic mild cognitive impairment: relationships to demographic variables and cognitive performance. J Clin Exp Neuropsychol 42:725-734

26. Avila J, Flowers A, Scott TM et al (2015) Daily activity abilities in mci, Alzheimer's disease, and healthy controls. GeroPsych (Bern) 28:191-200

Publisher's Note Springer Nature remains neutral with regard to jurisdictional claims in published maps and institutional affiliations. 